\title{
TO THE INFLUENCE OF MATERIAL DISPERSION ON THE INTERMODAL INTERFERENCE IN OPTICAL FIBRES
}

The comparison of computed and measured spectral dependence of intermodal interference in step-index optical fibre presented in this paper shows the influence of material dispersion on the intermodal interference in optical fibres. It is shown that the influence of material dispersion on intermodal interference is high enough to allow determination of the material dispersion difference of core and cladding of optical fibres.

\section{Introduction}

The meaning of the term "dispersion" is not determined unambiguously. In the papers devoted to physics problems it is used for expression of the fact that some quantity depends on the wavelength (frequency) of the light [1] or on the wavelength of the wave used at a quantity determination. This term is often used for expression of derivation of particular quantity on the wavelength. Most often the term dispersion is used for expression of derivation of material refractive index or derivation of phase velocity of the light on the wavelength - i.e. dispersion $d$ (taken as quantity) is determined by relation

$$
d=\partial n / \partial \lambda .
$$

For telecommunications purposes, the wavelength dependence of pulse transition time is important, so the critical parameter is not the phase velocity, but the group velocity of transmitted signal. Since the group velocity $v_{g}$ is equal to

$$
v_{g}=v+k \frac{\partial v}{\partial k}=v-\lambda \frac{\partial v}{\partial \lambda}
$$

where $v$ is the phase velocity and $k$ is the absolute value of the wave vector. So the spectral dependence of the pulse delay, determined with the group velocity dispersion, is characterised by the dispersion constant $[2,3]$

$$
D=\frac{\lambda}{c_{0}} \partial^{2} n / \partial \lambda^{2}
$$

where $c_{0}$ is the velocity of light in vacuum.

The phase velocity dispersion as well as the group velocity dispersion can be determined from the known wavelength dependence of the refractive index. There are a lot of sufficiently accurate methods for determination of the refractive index wavelength dependence in bulk materials. However, if it is to determine the dispersion of optical fibres, the problem arises. For determination of the core or cladding refractive index it is necessary to localise the lighting to an area smaller than the core diameter, that is, to the area with diameter of a few $\mu \mathrm{m}$. The sufficient illumination of so small area is possible, practically, only with lasers. But lasers are usually not wavelength-tuneable and tuneable only in a small region of wavelengths, respectively. To avoid the problems at determination of the material dispersion of optical fibres the investigation of intermodal interference of optical fibre can be used.

\section{Influence of dispersion on interference of modes}

As it is known, a study of the intermodal interference in optical fibres can be performed if the signal dependence given by interfering modes is detected with non-uniform sensitivity. The detection sensitivity in places where the phase of detecting modes is the same should differ from the places where their phases are opposite [4 - 7]. At such detection the influence of orthogonality of interfering modes is eliminated.

Taking into account the different phase constants of the modes, we can express the output of the quadratic detector located at the end of the fibre of length $\mathrm{z}$ as follows:

$$
s(z)=\int_{S} c(x, y) \cdot \sum_{i} \psi_{i}(x, y, z) \cdot \sum_{i} \psi_{i}^{*}(x, y, z) \cdot d x d y
$$

where $c(x, y)$ is the detector sensitivity, $\psi_{i}(x, y, z)$ are the functions describing the propagating modes and are equal to $\psi_{i, 0}(x, y)$. - $\exp \left(j \beta_{i} z\right)$, where $\psi_{i, 0}$ are the modal functions, $\beta_{i}$ are the phase constants of particular modes, $x$ and $y$ are the coordinates perpendicular to the direction of the propagation, $S$ is area on which the modal function is nonzero and "*” denotes complex conjugation. The expression (1) gives after some manipulation for the signal:

$$
\begin{aligned}
s(z) & =\int_{S} c(x, y) \sum_{k} \psi_{k, 0}(x, y) \cdot \psi_{k, 0}^{*}(x, y) \cdot d x d y+ \\
& +\int_{S} c(x, y) \sum_{l \neq k}^{0} \psi_{l, 0}(x, y) \cdot \psi_{k, 0}^{*}(x, y) \cdot \\
& \cdot \exp \left(j \cdot\left(\beta_{l}-\beta_{k}\right) z\right) \cdot d x d y .
\end{aligned}
$$

\footnotetext{
* ${ }^{1}$ Ivan Turek, ${ }^{2}$ Daniel Káčik, ${ }^{1}$ Ivan Martinček

${ }^{1}$ Department of Physics, University of Žilina

${ }^{2}$ Department of Telecommunications, University of Žilina
} 
The first term on the right side of Eq.(2) represents the sum of particular mode intensities and does not depend on the length of waveguide and only weakly depends on the wavelength (the dependence is only because of the dependence of the modal functions on wavelength).

The second term on the right side of Eq.(2) is the interference term. It is clear that while the sensitivity $c$ does not depend on coordinates its value is zero, because

$$
\int_{S} \psi_{l, 0}(x, y) \cdot \psi_{k, 0}^{*}(x, y) \cdot d x d y=0 \quad \text { for } \quad l \neq k
$$

At a suitable dependence of detector sensitivity on coordinates, the interference term can be nonzero. The interference term of two modes of a monochromatic light with wavelength $\lambda$, according the relation (2), is

$$
\begin{aligned}
\mathrm{s}(\mathrm{y}, \lambda) & =\int_{S} c(x, y) \cdot \psi_{l, 0}(x, y) \cdot \psi_{k, 0}^{*}(x, y) \cdot \\
& \cdot \exp \left(j \cdot\left(\beta_{l}-\beta_{k}\right) z\right) \cdot d x d y
\end{aligned}
$$

or

$$
s_{\text {int }}(z, \lambda)=s_{0} \cdot \cos \left(\Delta \beta_{l, k}(\lambda) z\right)
$$

where $\Delta \beta_{l, k}(\lambda)=\Delta \beta_{l}(\lambda)-\Delta \beta_{k}(\lambda)$, indexes $l, k$ correspond to interfering modes and

$$
s_{0}=\int_{S} c(x, y) \cdot \psi_{l, 0}(x, y) \cdot \psi_{k, 0}^{*}(x, y) \cdot d x d y .
$$

If we investigate intermodal interference of modes in the light of finite spectral width $\Delta \lambda$, the interference term (4) should be modified. As waves with different frequencies participate in the interference of mode, the term has to be rewritten in the form

$$
s_{\text {int }}(l, \lambda)=s_{0} \cdot \int_{\Delta \lambda} \cos \left(\Delta \beta_{l, k}(\lambda) \cdot z\right) \cdot \zeta(\lambda) \cdot d \lambda
$$

where $z$ is the length of the fibre and $\zeta(\lambda)$ is the spectral density of the light. As $\zeta$ depends on $\lambda$ only slightly, the Eq. $(5, a)$ can be rewritten into

$$
s_{i n t}(l, \lambda)=s_{0} \bar{\zeta} \cdot \int_{\Delta \lambda} \cos \left(\Delta \beta_{l, k}(\lambda) \cdot z\right) \cdot d \lambda
$$

where $\bar{\zeta}$ is the average value of $\zeta$ in the region $\Delta \lambda$.

In Fig.1. the typical curve of transmission function of fibre with nonzero interfering term is shown. As it can be seen from the dependence shown in this figure, the spectral dependencies of experimental observing curves have a character corresponding to the expression (5).

The harmonic character of interference term given by equation (4) (or its modified form (5)) allows to separate the interference term from the measured transmission function. The spectral dependence of interference term obtained from the measured transmission function drawn in Fig. 1. is presented in Fig. 2. In this figure there is also calculated spectral dependence of inter- ference term of the first two modes. It was calculated for such parameters of fibre (values of refractive index of core and cladding, radius of core and length of the fibre) for which the interference centre $\lambda_{0}[8]$ corresponds to the measured interference centre and the periodicity of the dependence is in the maximal agreement with the measured one.

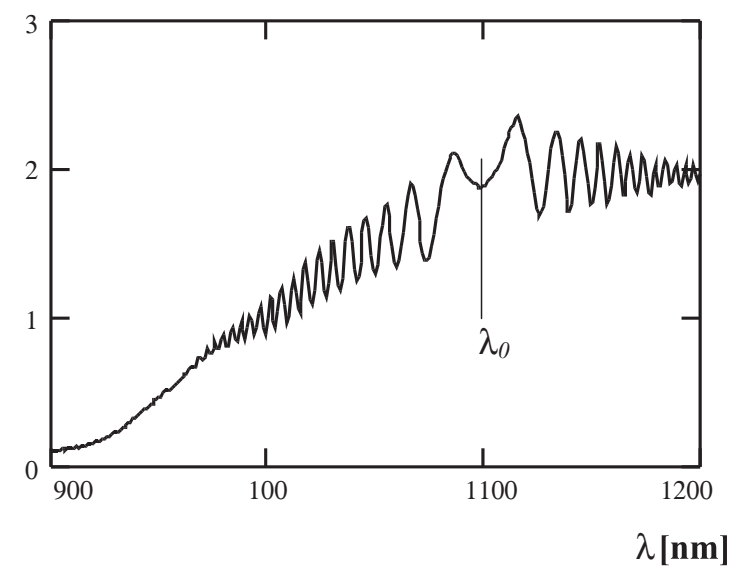

Fig. 1. Spectral dependence of transmission function (in arbitrary units) with significant interference term measured on Siemens fibre.

It can be seen from comparison of these dependencies that their character is the same but the periodicity of the calculated dependence does not correspond to the measured one.

The difference of measured and calculated curves drawn in Fig. 2 is caused by the fact that the phase constants $\beta_{l}$ and $\beta_{k}$ (which determinate the phase difference of interfering modes) depend on the wavelength not only explicitly, but also through the wavelength dependence of refractive index of core and cladding. This means that $\Delta \beta$ (and the periodicity of signal too) is a function of material dispersion of the core and the cladding. So the interference of modes can be used as an information source of fibre material dispersion.

It follows from the previous that the interference of modes depends on difference of material dispersion of fibre's core and cladding, but not on the dispersion itself. The core or the cladding of the fibre is often made from pure silica (for which the dispersion is very well known), so the material dispersion difference, which can be evaluated by studying the intermodal interference, allows to determine the material dispersion of the non-silica material built in the optical fibre.

The following "model example" illustrates the influence of material dispersion on the intermodal interference and possible use of intermodal interference for its determination.

When it is assumed that the wavelength dependence of refractive index of core is the same as it is in pure silica, i.e.

$$
n_{c o}=1.4471+0.014396\left(\lambda-1.1 \cdot 10^{-6} \mathrm{~m}\right) / 1.1 \cdot 10^{-6} \mathrm{~m}
$$




\section{kam NikCole}

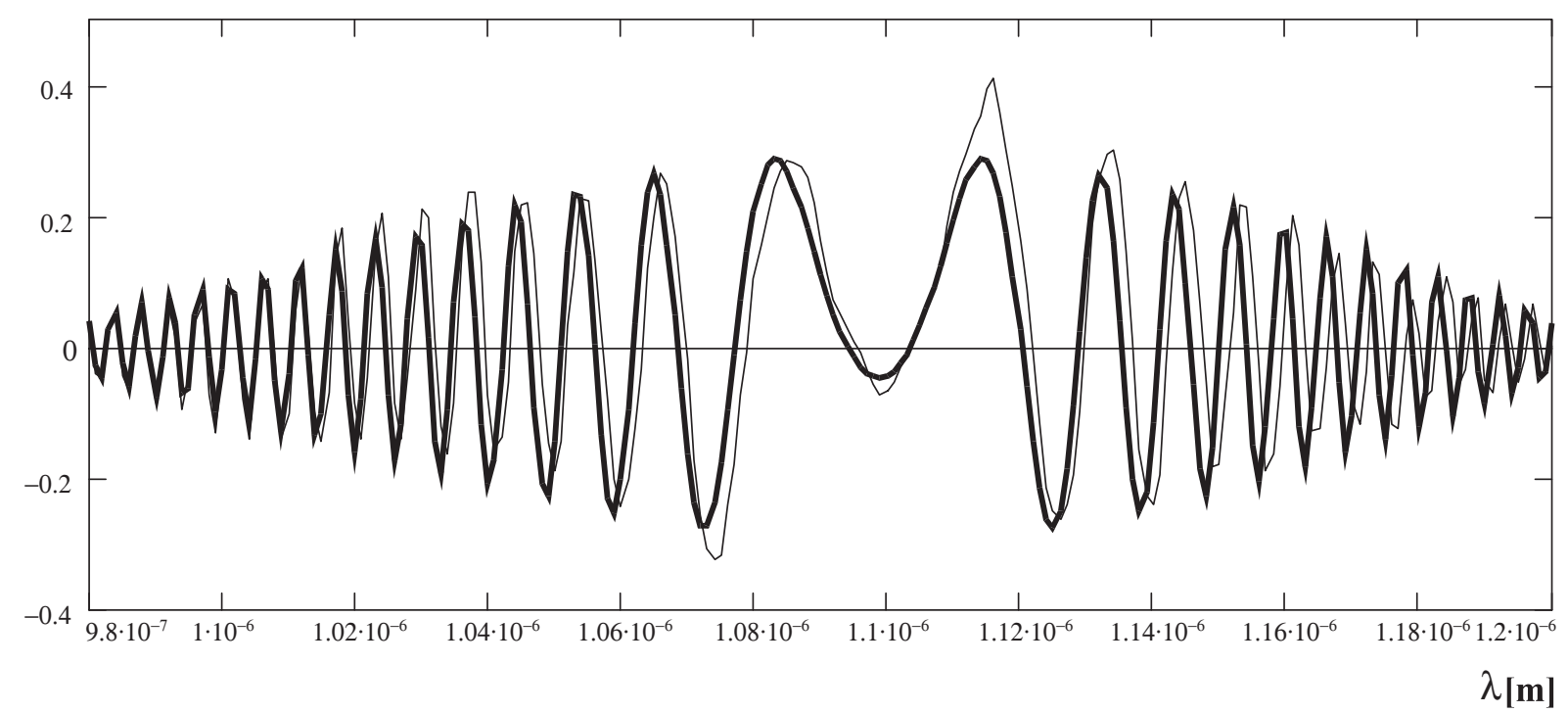

Fig. 2. The comparison between measured __ and computed interference term with constant refractive indexes $\longrightarrow$. Values are plotted in arbitrary units.

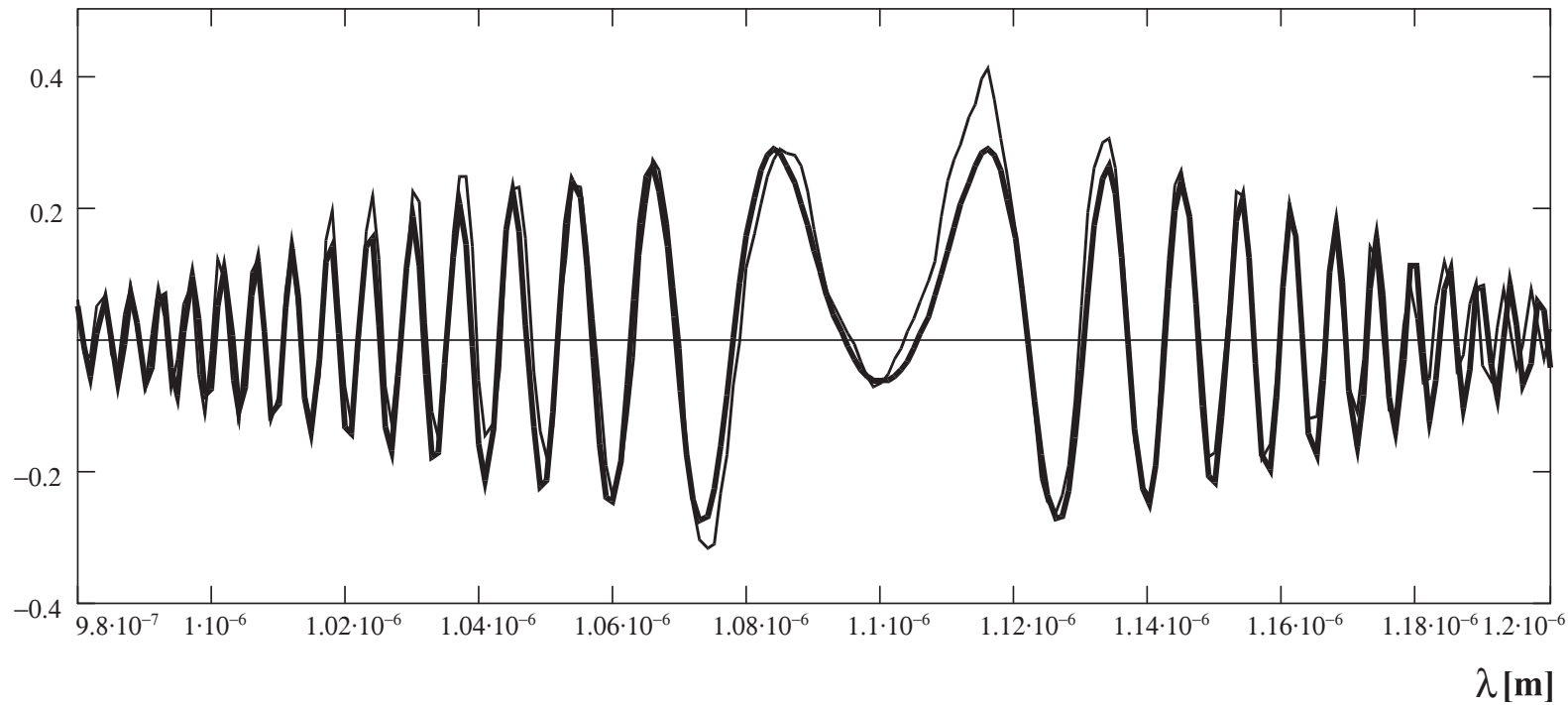

Fig. 3. The comparison between measured — and computed interference term with linear dependence of dispersion - . Values are plotted in arbitrary units.

and that the refractive index of cladding is

$$
n_{c l}=1.443299-0.01445\left(\lambda-1.1 \cdot 10^{-6} \mathrm{~m}\right) / 1.1 \cdot 10^{-6} \mathrm{~m}
$$

the dependence of phase constants difference of the first and second modes calculated for weakly-guiding step-index optical fibre of length $0.72 \mathrm{~m}$, gives spectral dependence of interference term which is drawn in Fig.3.

The difference of the interference term calculated at that assumption and the measured dependence is smaller than error of measurement in the area of the wavelengths 0.8 to $1.16 \mu \mathrm{m}$. In the area close to $1.2 \mu \mathrm{m}$ a difference between computed and measured dependencies is seen. This difference can be eliminated introducing a quadratic dependence of cladding refractive index. When the refractive index of cladding in area from 1.13 to $1.2 \mu \mathrm{m}$ is assumed to be greater than that one which was taken into account for the curve given in Fig. 3. by the value equal to $3.0510^{8}\left(\lambda-1.13 \cdot 10^{-6}\right)^{2}$, then practically full correspondence of the measured and calculated dependencies is obtained in all investigated region (Fig. 4.). 


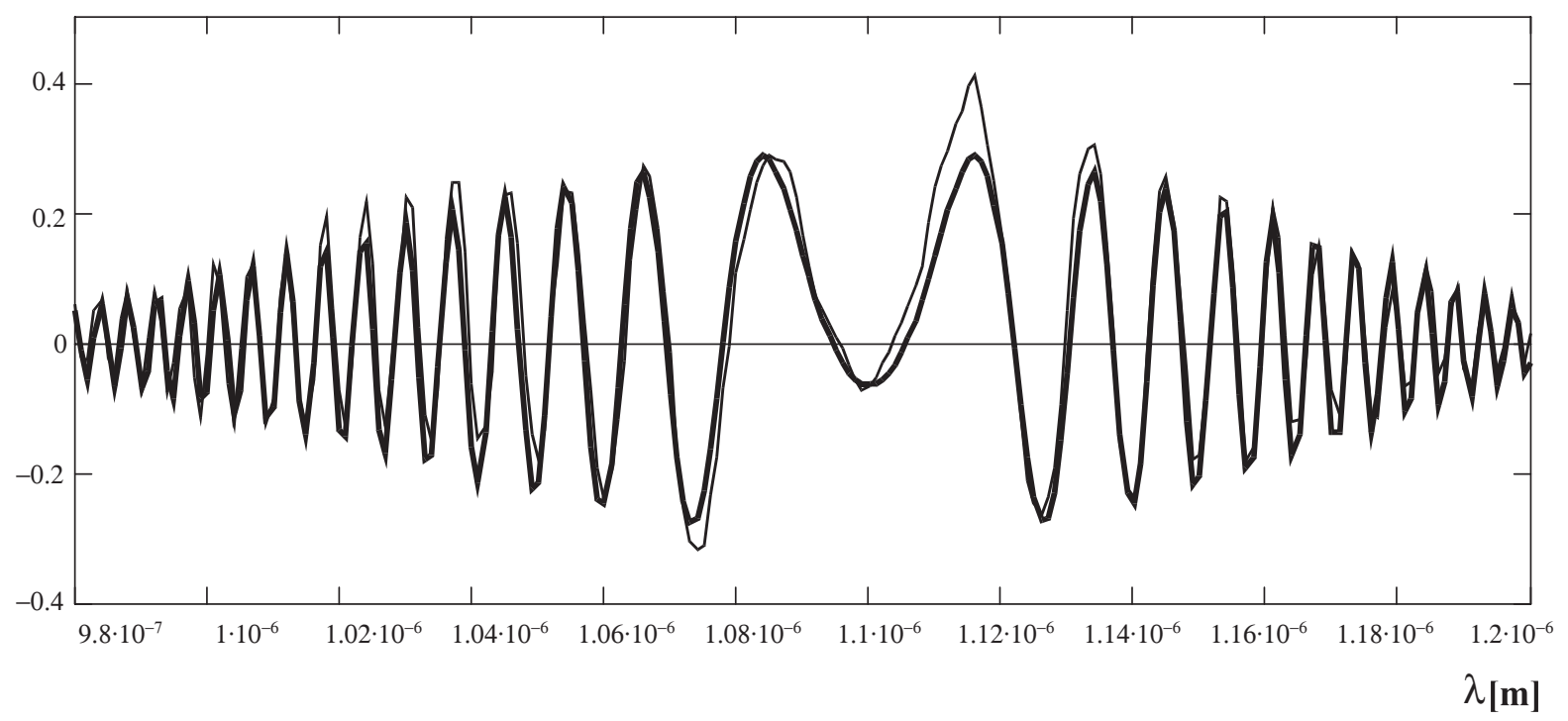

Fig. 4. The comparison between measured _ and computed interference term with linear and quadratic dependence of refractive index being a function of wavelength (group velocity dispersion)

Values are plotted in arbitrary units.

\section{Conclusion}

The presented example shows that spectral dependence of interference term reflects the wavelength dependence of refractive index in a very sensitive way. This sensitivity is so high that the study of intermodal interference can be used not only for examination of geometrical parameters of fibres [7, 8] (radius of core, refractive index profile) but also for determination of material dispersion of investigated fibres.

Technical equipment used in our laboratory allows to measure spectral dependencies with precision of $1 \mathrm{~nm}$ of the wavelengths. In the previous paragraph it was showed that such precision allows to observe influence of spectral dependence of interference of modes and to determine its derivation on the wavelength. If the wavelength region in which the interference term is registered is wide enough, it allows also to estimate the second derivation of the difference of indices of the core and the cladding.

Nowadays, in good laboratories there is no problem to determine spectral dependencies with accuracy higher than $0.1 \mathrm{~nm}$. Such precision allows to determine the first and the second derivation of refractive indices also if intermodal interference is observed in an area of wavelengths smaller than the area, which was necessary in our measurement in the presented model example.

\section{References}

[1] BORN, M., WOLF, E.: Principles of Optics, Cambridge University Press, 2002, p. 95.

[2] KEISER, G.: Optical fiber communications, McGraw-Hill, 2000, p. 107.

[3] DADO, M., TUREK, I., ŠTELINA, J., BITTERER, L., TUREK, S., GROLMUS, E., STIBOR, P.: Kapitoly z optiky pre technikov, Žilinská univerzita, 1998, p. 316.

[4] TUREK, I., MARTINČEK, I., STRÁNSKY, R.: “Interference of modes in optical fibers," Opt.Eng., vol. 39, 1304-1309, 2000

[5] POSEY, R., PHILLIPS, L., DIGGS, D., SHARMA, A.: "LP01-LP02 interference using a spectrally extended light source: measurement of the non-step-refractive-index profile of optical fibers, "Opt. Lett., vol. 21, 1357-1359, 1996

[6] HLUBINA, P.: "Spectral detection schemes for the low-coherence based two-mode fibre waveguide sensors", Poceedings SPIE, vol. 2510 (Fiber Optic and Laser Sensors XIII, Munich 1995), pp. 213-223.

[7] ARCHAMBAULT, J. L., BLACK, R. J., BURES, J., GONTHIER, F., LACROIX, S., SARAVANOS C.: "Fiber core profile characterization by measuring group velocity equalization wavelengths," IEEE Photon. Technol. Lett., vol. 3, 351-353, 1991

[8] MARTINČEK, I., TUREK, I., DADO, M., GRONDŽÁK, K., ČERNICKÝ, S.: "Interferencia módov v optických vláknach", Komunikácie/Communications 2/2000. 